\title{
Positive Solutions for a Boundary Value Problem with a Derivative Argument ${ }^{*}$
}

\author{
Bo Sun \\ School of Statistics and Mathematics, Central University of Finance and Economics, Beijing 100081, China \\ Email: sunbo19830328@163.com
}

Received 2013

\begin{abstract}
In this paper, several existence results of multiple positive solutions are obtained for a boundary value problem with $\mathrm{p}$-Laplacian, by applying a fixed point theorem in cones. The interesting point is that the nonlinear term $\mathrm{f}$ is involved with the first-order derivative explicitly.
\end{abstract}

Keywords: Positive Solutions; Fixed Point Theorem; Cone; Boundary Value Problem; P-Laplacian

\section{Introduction}

In this paper, we will consider the existence of multiple positive solutions for the following one-dimensional p-Laplacian, multi-point boundary value problem

$$
\begin{array}{r}
\left(\phi_{p}\left(u^{\prime}(t)\right)\right)^{\prime}+f\left(t, u(t), u^{\prime}(t)\right)=0, \quad t \in(0,1), \\
u^{\prime}(0)=\sum_{i=1}^{n} \alpha_{i} u^{\prime}\left(\xi_{i}\right), \quad u(1)=\sum_{i=1}^{n} \beta_{i} u\left(\xi_{i}\right),
\end{array}
$$

where $\phi_{p}(s)=|s|^{p-2} s, \quad p>1, \quad \xi_{i} \in(0,1)$ with $0<\xi_{1}<\xi_{2}$ $<\cdots<\xi_{n}<1$ and $\alpha_{i}, \beta_{i}$, $f$ satisfy,

$(H 1): 0 \leq \alpha_{i}, \beta_{i}<1(i=1,2, \ldots, n-2)$ satisfy

$$
0 \leq \sum_{i=1}^{n} \alpha_{i}<1, \quad 0 \leq \sum_{i=1}^{n} \beta_{i}<1
$$

$(H 2): \quad f(t, u, v) \in C([0,1] \times[0,+\infty) \times R \rightarrow[0,+\infty))$, and $f(t, 0,0) \neq 0$ on any subinterval of $(0,1)$.

$(H 3): f\left(t, u_{1}, v_{1}\right) \leq f\left(t, u_{2}, v_{2}\right)$

for any $0 \leq t \leq 1,0 \leq u_{1} \leq u_{2}, \quad v_{1} \leq v_{2}$;

(H4) : $f(t, u, v) \leq g(t)|u|^{a}+h(t)|v|^{b}+j(t)$, for $0 \leq t \leq 1, \quad u \geq 0$, where $g(t), h(t), j(t) \in L^{1}[0,1]$, $0 \leq a, b<p-1$.

There is much current interest in questions of multiple positive solutions of boundary value problems, and the existence and multiplicity of positive solutions for linear and nonlinear multipoint boundary value problems have been widely studied by many authors, one may see [1-9] and relevant literatures.

*This work was supported by the National Natural Science Foundation of China (NO.11126245), Youth PhD Development Fund of Central University of Finance and Economics 121 Talent Cultivation Project (NO.QBJZH201004) and Discipline Construction Fund of Central University of Finance and Economics.
Among the substantial number of works dealing with nonlinear differential equations we mention the boundary value problem (1.1) and (1.2). We emphasize that the nonlinear term $f$ is involved with the first-order derivative explicitly in this problem.

\section{Definition and Theoretical Foundation}

In this section, we give definition and theoretical foundation in this paper.

Definition 2.1. Let $E$ be a real Banach space over R. A nonempty closed set $P \subset E$ is said to be a cone provided that

(i) $a u+b v \in P$ for all $u, v \in P$ and all $a \geq 0$, $b \geq 0$, and

(ii) $u,-u \in P$ imply $u=0$.

To prove our main results, we need the following fixed point theorem in cones.

Theorem 2.1. [7] Let $K$ be a cone in a Banach space $X$.

Let $D$ be an open bounded subset of $X$ with

$$
D_{k}=D \cap K \neq \varnothing \text {. }
$$

Assume that $A: \overline{D_{k}} \rightarrow K$ is completely continuous such that $x \neq A x$ for $x \in \partial D_{k}$.

Then the following results hold:

(S1) If $\|A\| x\|\leq\| x, x \in \partial D_{k}$, then $i_{k}\left(A, D_{k}\right)=1$.

(S2) If there exists $e \in K \backslash\{0\}$ such that $x \neq A x+\lambda e$ for

all $x \in \partial D_{k}$ and all $\lambda>0$, then $i_{k}\left(A, D_{k}\right)=0$.

(S3) Let $U$ be open in $X$ such that $\bar{U} \subset D_{k}$.

If $i_{k}\left(A, D_{k}\right)=1$ and $i_{k}\left(A, U_{k}\right)=0$, then $A$ has a fixed point in $D_{k} \backslash \overline{U_{k}}$. 
The same result holds if $i_{k}\left(A, D_{k}\right)=0$ and $i_{k}\left(A, U_{k}\right)=1$.

\section{Preliminaries}

Let the Banach space $E=C^{1}[0,1]$ be endowed with the norm $\| u \mid:=\max \left\{\max |u(t)|, \max \left|u^{\prime}(t)\right|\right\}$.

We denote $E_{+}=C_{+}^{1}[0,1\}=\{u \in E \mid u(t) \geq 0, t \in[0,1]\}$, and define the cone $P \subset E$ by

$P=\{u \in E \mid u(t) \geq 0, u$ is concave on $[0,1]$ and satisfy $(1.2)\}$.

Throughout, it is assumed that $(H 1)-(H 4)$ hold.

Lemma 3.1. Suppose $y \in C^{1}[0,1]$ with

$\left(\phi_{p}\left(y^{\prime}(t)\right)\right)^{\prime} \in C^{1}[0,1]$

satisfies,

$$
\begin{aligned}
& -\left(\phi_{p}\left(y^{\prime}(t)\right)\right)^{\prime} \geq 0, \quad t \in(0,1), \\
& y^{\prime}(0)=\sum_{i=1}^{n} \alpha_{i} y^{\prime}\left(\xi_{i}\right), \quad y(1)=\sum_{i=1}^{n} \beta_{i} y\left(\xi_{i}\right) .
\end{aligned}
$$

Then, $y(t)$ is concave and $y(t) \geq 0$, i.e., $y \in P$, and $y^{\prime}(t) \leq 0$ on $[0,1]$.

Proof. The proof is very easy since

$$
0 \leq \sum_{i=1}^{n-2} \alpha_{i}, \sum_{i=1}^{n-2} \beta_{i}<1
$$

so we omit it here.

For any $x \in C_{+}[0,1]$, suppose $u$ is a solution of the problem

$$
\left\{\begin{array}{l}
\left(\phi_{p}\left(u^{\prime}\right)\right)^{\prime}+f\left(t, x, x^{\prime}\right)=0,0 \leq t \leq 1, \\
u^{\prime}(0)=\sum_{i=1}^{n-2} \alpha_{i} u^{\prime}\left(\xi_{i}\right), u(1)=\sum_{i=1}^{n-2} \beta_{i} u\left(\xi_{i}\right),
\end{array}\right.
$$

then we have

$$
\begin{aligned}
& u^{\prime}(t)= \phi_{p}^{-1}\left(W_{x}-\int_{0}^{t} f\left(s, x(s), x^{\prime}(s)\right) d s\right), \\
& u(t)=-\frac{\sum_{i=1}^{n-2} \beta_{i} \int_{\xi_{i}}^{1} \phi_{p}^{-1}\left(W_{x}-\int_{0}^{s} f\left(r, x(r), x^{\prime}(r)\right) d r\right) d s}{1-\sum_{i=1}^{n-2} \beta_{i}} \\
&-\int_{t}^{1} \phi_{p}^{-1}\left(W_{x}-\int_{0}^{s} f\left(r, x(r), x^{\prime}(r)\right) d r\right) d s,
\end{aligned}
$$

where $W_{x}$ satisfy

$$
\phi_{p}^{-1}\left(W_{x}\right)=\sum_{i=1}^{n-2} \alpha_{i} \phi_{p}^{-1}\left(W_{x}-\int_{0}^{\xi_{i}} f\left(r, x(r), x^{\prime}(r)\right) d r\right) .
$$

Lemma 3.2. $x \in C_{+}^{1}[0,1]$, there exists a unique $W_{x}$ satisfies

(3.1) and

$$
W_{x} \in\left[-\frac{\phi_{p}\left(\sum_{i=1}^{n} \alpha_{i}\right)}{1-\phi_{p}\left(\sum_{i=1}^{n} \alpha_{i}\right)} \int_{0}^{1} f\left(r, x(r), x^{\prime}(r)\right) d r, 0\right] .
$$

Proof. The proof is very similar to the proof of Lem- ma 2.2 in [8], so we omit it here.

Lemma 3.3. For $u \in P$, there exists a constant $\gamma_{1}$,

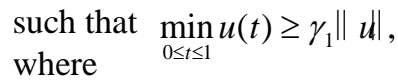

$$
\gamma_{1}=\min \left\{\frac{\sum_{i=1}^{n} \beta_{i}\left(1-\xi_{i}\right)}{1-\sum_{i=1}^{n} \beta_{i}}\left(\frac{1}{\lambda}\right)^{\frac{1}{p-1},} \frac{\sum_{i=1}^{n} \beta_{i}\left(1-\xi_{i}\right)}{1-\sum_{i=1}^{n} \beta_{i} \xi_{i}}\right\},
$$

and $\lambda>1$ is a constant such that

$$
0<\xi_{1}<2 \xi_{1}<3 \xi_{1}<\cdots<(\lambda-1) \xi_{1}<1<\lambda \xi_{1} \text {. }
$$

Proof. By lemma 3.1, we have

$$
\min _{0 \leq t \leq 1} u(t)=u(1), \quad\|u\|=\max \left\{u(0),-u^{\prime}(1)\right\} .
$$

For $u \in P$, by the concavity of $u$, we have

$$
\frac{u\left(\xi_{i}\right)-u(1)}{1-\xi_{i}} \geq \frac{u(0)-u(1)}{1},
$$

that is $u\left(\xi_{i}\right)-\xi_{i} u(1) \geq\left(1-\xi_{i}\right) u(0)$,

to combine with the boundary condition (1.2) we have

$$
\sum_{i=1}^{n-2} \beta_{i} u\left(\xi_{i}\right)-\sum_{i=1}^{n-2} \beta_{i} \xi_{i} u(1) \geq \sum_{i=1}^{n-2} \beta_{i}\left(1-\xi_{i}\right) u(0)
$$

so

$$
\min _{0 \leq t \leq 1} u(t)=u(1) \geq \frac{\sum_{i=1}^{n-2} \beta_{i}\left(1-\xi_{i}\right)}{1-\sum_{i=1}^{n-2} \beta_{i} \xi_{i}} u(0) .
$$

By $\left(H_{3}\right)$ and lemma 3.2, we have

$$
\begin{aligned}
& \lambda\left(-W_{x}+\int_{0}^{\xi_{1}} f\left(r, x(r), x^{\prime}(r)\right) d r\right) \\
& \geq\left(-W_{x}+\int_{0}^{\xi_{1}} f\left(r, x(r), x^{\prime}(r)\right) d r\right) \\
& +\left(-W_{x}+\int_{\xi_{1}}^{2 \xi_{1}} f\left(r, x(r), x^{\prime}(r)\right) d r\right) \\
& +\cdots\left(+-W_{x}+\int_{(\lambda-1) \xi_{1}}^{\lambda \xi_{1}}\left(f, r(x) r\left(x^{\prime}\right)\right) d r\right. \\
& =-\lambda W_{x}+\int_{0}^{\lambda \xi_{1}} f\left(r, x(r), x^{\prime}(r)\right) d r \\
& \geq-W_{x}+\int_{0}^{1} f\left(r, x(r), x^{\prime}(r)\right) d r,
\end{aligned}
$$

therefore,

$$
\begin{gathered}
-W_{x}+\int_{0}^{\xi_{1}} f\left(r, x(r), x^{\prime}(r)\right) d r \\
\geq \frac{1}{\lambda}\left(-W_{x}+\int_{0}^{1} f\left(r, x(r), x^{\prime}(r)\right) d r\right) .
\end{gathered}
$$

On the other hand,

$$
=\frac{\sum_{i=1}^{\min } u(t)=u(1)}{\sum_{i} \int_{\xi_{i}}^{1} \phi_{P}^{-1}\left(-W_{x}+\int_{0}^{s} f\left(r, x(r), x^{\prime}(r)\right) d r\right) d s}
$$




$$
\begin{aligned}
& \geq \frac{\sum_{i=1}^{n} \beta_{i}\left(1-\xi_{i}\right)}{1-\sum_{i=1}^{n} \beta_{i}} \phi_{P}^{-1}\left(-W_{x}+\int_{0}^{\xi_{1}} f\left(r, x(r), x^{\prime}(r)\right) d r\right) \\
& \geq \frac{\sum_{i=1}^{n} \beta_{i}\left(1-\xi_{i}\right)}{1-\sum_{i=1}^{n} \beta_{i}}\left(\frac{1}{\lambda}\right)^{\frac{1}{p-1}} \phi_{P}^{-1}\left(-W_{x}+\int_{0}^{1} f\left(r, x(r), x^{\prime}(r)\right) d r\right) \\
& =\frac{\sum_{i=1}^{n} \beta_{i}\left(1-\xi_{i}\right)}{1-\sum_{i=1}^{n} \beta_{i}}\left(\frac{1}{\lambda}\right)^{\frac{1}{p-1}}\left(-u^{\prime}(1)\right) \text {. }
\end{aligned}
$$

From the discussion above, choose $\gamma_{1}$ by (3.2), we can obtain the lemma 3.3 is proved.

Lemma 3.4. For $u \in P$, there exists a constant $N>0$, such that $\max ^{\prime}\left|u^{\prime}(t)\right| \leq N$.

Proof. For $u \in P^{0 \leq t \leq b y}$ the concavity of $u$ and lemma 3.1, there is

$$
\begin{aligned}
& u(t)-u(1) \leq-u^{\prime}(1) \\
& =\max _{0 \leq t \leq 1}\left|u^{\prime}(t)\right|, \quad t \in[0,1] .
\end{aligned}
$$

Taking into account that $u(1)=\sum_{i=1}^{n} \beta_{i} u\left(\xi_{i}\right)$, we have $\max _{0 \leq t \leq 1}|u(t)| \leq\left(\sum_{i=1}^{n} \beta_{i}\right) \max _{0 \leq t \leq 1}|u(t)|+\max _{0 \leq t \leq 1}\left|u^{\prime}(t)\right|$.

So,

$$
\left(1-\sum_{i=1}^{n} \beta_{i}\right) \max _{0 \leq t \leq 1}|u(t)| \leq \max _{0 \leq t \leq 1}\left|u^{\prime}(t)\right| .
$$

And because

$$
u^{\prime}(t)=\phi_{p}^{-1}\left(W_{x}-\int_{0}^{t} f\left(s, x(s), x^{\prime}(s)\right) d s\right),
$$

in view of (H3), we have

$$
\begin{aligned}
& \left(\left|u^{\prime}(t)\right|\right)^{p-1}
\end{aligned}
$$

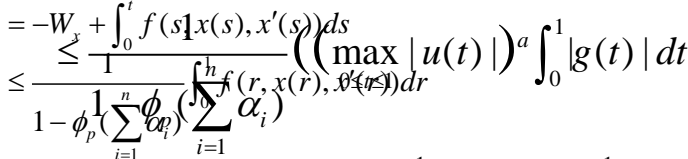

$$
\begin{aligned}
& \left.+\left(\max _{0 \leq t \leq 1}\left|u^{\prime}(t)\right|\right)^{b} \int_{0}^{1} h(t)\left|d t+\int_{0}^{1}\right| j(t) \mid d t\right) \\
& \leq \frac{1}{1-\phi_{p}\left(\sum_{i=1}^{n} \alpha_{i}\right)}\left(\frac{1}{\left(1-\sum_{i=1}^{n} \beta_{i}\right)^{a}}\left(\max _{0 \leq t \leq 1}\left|u^{\prime}(t)\right|\right)^{a} \int_{0}^{1}|g(t)| d t\right. \\
& \left.+\left(\max _{0 \leq t \leq 1}\left|u^{\prime}(t)\right|\right)^{b} \int_{0}^{1} h(t)\left|d t+\int_{0}^{1}\right| j(t) \mid d t\right) .
\end{aligned}
$$

Since $0 \leq a, b<p-1$, we have $\max |u(t)|$ is bounded. Therefore, there exists a constant $N>0$, such that lemma 3.4 is proved.

For notational convenience, we denote

$$
\begin{aligned}
& \theta=\frac{\phi_{p}\left(\sum_{i=1}^{n} \alpha_{i}\right)}{1-\phi_{p}\left(\sum_{i=1}^{n} \alpha_{i}\right)}, \eta=\frac{1}{1-\phi_{p}\left(\sum_{i=1}^{n} \alpha_{i}\right)}, \\
& \frac{1}{l}=\max \left\{\frac{p-1}{p}\left(\frac{\sum_{i=1}^{n} \beta_{i}}{1-\sum_{i=1}^{n} \beta_{i}}\left(\eta^{\frac{p}{p-1}}-\left(\xi_{i}+\theta\right)^{\frac{p}{p-1}}\right)+\left(\eta^{\frac{p}{p-1}}-\theta^{\frac{p}{p-1}}\right)\right), \eta^{\frac{1}{p-1}}\right\}, \\
& \frac{1}{L}=\frac{p-1}{p}\left(1-\sum_{i=1}^{n} \beta_{i}\right)^{-1} \sum_{i=1}^{n} \beta_{i}\left(1-\xi_{i}^{\frac{p}{p-1}}\right),
\end{aligned}
$$

then define

$$
K=\left\{u \mid u \in E, \min _{0 \leq t \leq 1} u(t) \geq \gamma\|u\|,-N \leq u^{\prime} \leq 0,\right\}
$$
where $\gamma=\gamma_{1} \gamma_{2}, \gamma_{1}$ is defined by (3.2), $\gamma_{2}=\frac{l}{L}, N$
is defined in lemma 3.4.

It is easy to obtain that, $0<l, L<\infty$ and $L \gamma=L \gamma_{1} \gamma_{2}=\gamma_{1} l<l$.

We define an operator $T: K \rightarrow E$ by

$$
\begin{gathered}
(T u)(t)=\frac{-\sum_{i=1}^{n} \beta_{i} \int_{\xi_{i}}^{1} \phi_{p}^{-1}\left(W_{u}-\int_{0}^{s} f\left(r, u(r), u^{\prime}(r)\right) d r\right) d s}{1-\sum_{i=1}^{n} \beta_{i}} \\
-\int_{t}^{1} \phi_{p}^{-1}\left(W_{u}-\int_{0}^{s} f\left(r, u(r), u^{\prime}(r)\right) d r\right) d s,
\end{gathered}
$$

where $W_{u}$ is defined in (3.1). Then a standard argument shows that $T: K \rightarrow E$ is completely continuous. Define

$$
\begin{aligned}
K_{N} & =\{x \in K \mid\|x\|<N\}, \\
\Omega_{N} & =\left\{x \in K \mid \min _{0 \leq t \leq 1} x(t)<\gamma N,-N<x^{\prime}(t)<0\right\} \\
& =\left\{x\left|x \in C^{1}[0,1], x \geq 0, \gamma \| x\right|\right. \\
& \left.\leq \min _{0 \leq t \leq 1} x(t)<\gamma N,-N<x^{\prime}(t)<0\right\} .
\end{aligned}
$$

Lemma 3.5. [9] $\Omega_{N}$ has the following properties: $\Omega_{N}$ is open relative to $K$;

$$
K_{\gamma N} \subset \Omega_{N} \subset K_{N} ;
$$

$x \in \partial \Omega_{N}$ if and only if $\min x(t)=\gamma N, \min x^{\prime}(t)=-N$;

If $x \in \partial \Omega_{N}$, then $\gamma^{0} \mathcal{N}^{\prime \leq 1} \leq x(t) \leq N, \stackrel{0 \leq t \leq 1}{N} \leq x^{\prime}(t) \leq 0$ for $t \in[0,1]$.

For notational convenience, we introduce the following notations

$$
\begin{aligned}
& f_{-N, 0}^{\gamma N, N}=\min \left\{\frac{f(t, u, v)}{\phi_{p}(N)}: t \in[0,1], u \in[\gamma N, N], v \in[-N, 0]\right\}, \\
& f_{-N, 0}^{0, N}=\max \left\{\frac{f(t, u, v)}{\phi_{p}(N)}: t \in[0,1], u \in[0, N], v \in[-N, 0]\right\}, \\
& f_{-N, 0}^{\delta}=\lim _{u \rightarrow \alpha} \max \left\{\frac{f(t, u, v)}{\phi_{p}(u)}: t \in[0,1], v \in[-N, 0]\right\}, \\
& f_{\delta}^{-N, 0}=\lim _{u \rightarrow \alpha} \min \left\{\frac{f(t, u, v)}{\phi_{p}(u)}: t \in[0,1], v \in[-N, 0]\right\}, \\
& \left(\delta=\infty \text { or } 0^{+}\right) .
\end{aligned}
$$


Lemma 3.6. If $f$ satisfies

$$
f_{-N, 0}^{0, N}<\phi_{p}(l),
$$

then $i_{k}\left(T, K_{N}\right)=1$.

Proof. From the definition of $\mathrm{T}$ and by (3.4), for $u(t) \in \partial K_{N}$,

$$
\begin{gathered}
\quad \frac{\sum_{i=1}^{n} \beta_{i} \int_{\xi_{i}}^{1} \phi_{p}^{-1}\left(-W_{u}+\int_{0}^{s} f\left(r, u(r), u^{\prime}(r)\right) d r\right) d s}{1-\sum_{i=1}^{n} \beta_{i}} \\
\quad+\int_{0}^{1} \phi_{q}\left(-W_{u}+\int_{0}^{s} f\left(r, u(r), u^{\prime}(r)\right) d r\right) d s \\
<\operatorname{lN} \frac{p-1}{p}\left(\frac{\sum_{i=1}^{n} \beta_{i}}{1-\sum_{i=1}^{n} \beta_{i}}\left(\eta^{\frac{p}{p-1}}-\left(\xi_{i}+\theta\right)^{\frac{p}{p-1}}\right)+\left(\eta^{\frac{p}{p-1}}-\theta^{\frac{p}{p-1}}\right)\right) \leq N,
\end{gathered}
$$

and

$$
\begin{aligned}
\left|(T u)^{\prime}(t)\right| & =\phi_{p}^{-1}\left(-w_{u}+\int_{0}^{t} f\left(r, u(r), u^{\prime}(r)\right) d r\right) \\
& <I N \eta^{\frac{1}{P-1}} \leq N .
\end{aligned}
$$

So, we have $\|\mathbb{T} u\| k \| u$ for $u(t) \in \partial K_{N}$. By Theorem 2.1, we have $i_{k}\left(T, K_{N}\right)=1$.

Lemma 3.7. If $f$ satisfies

$$
f_{-N, 0}^{\gamma N, N}>\phi_{p}(L \gamma)
$$

then $i_{k}\left(T, \Omega_{N}\right)=0$.

Proof. Let $e(t) \equiv 1$ for $t \in[0,1]$. Then $e \in \partial K_{1}$. we claim that

$$
u \neq T u+\rho e, \quad u \in \partial \Omega_{N}, \quad \rho \geq 0 .
$$

In fact, if the assumption fails, there exist $u_{0} \in \partial \Omega_{N}$ and $\rho_{0} \geq 0$ such that $u_{0}=T u_{0}+\rho_{0} e$.

From the definition of $\mathrm{T}$ and by lemma 3.5, for $t \in[0,1]$, we have

$$
\begin{gathered}
u_{0}(t)=T u_{0}(t)+\rho_{0} e u_{0}(t)=T u_{0}(t)+\rho_{0} e \\
\geq \frac{\sum_{i=1}^{n} \beta_{i} \int_{\xi_{i}}^{1} \phi_{q}\left(-W_{u_{0}}+\int_{0}^{s} f\left(r, u_{0}(r), u_{0}{ }^{\prime}(r)\right) d r\right) d s}{1-\sum_{i=1}^{n} \beta_{i}}+\rho_{0} \\
>\frac{\sum_{i=1}^{n} \beta_{i}}{1-\sum_{i=1}^{n} \beta_{i}} \frac{p-1}{p} L \gamma N\left(1-\xi_{i}^{\frac{p}{p-1}}\right)+\rho_{0} \\
>\gamma N+\rho_{0} .
\end{gathered}
$$

This implies $\gamma N>\gamma N+\lambda_{0}$, which is a contradiction.

So, by Theorem 2.1, we obtain $i_{k}\left(T, \Omega_{N}\right)=0$.

\section{Multiple Positive Solutions to (1.1), (1.2)}

Our main results are as follows.

Theorem 4.1. Suppose $(H 1)-(H 4)$ hold, and

(H5): there exist $\rho_{1}, \rho_{2}, \rho_{3} \in(0, N)$, with $\rho_{1}<\gamma \rho_{2}<\rho_{2}<\rho_{3}$ such that

$$
f_{-\rho_{1}, 0}^{0, \rho_{1}}<\phi_{p}(l), \quad f_{-\rho_{2}, 0}^{\gamma \rho_{2}, \rho_{2}}>\phi_{p}(L \gamma), \quad f_{-\rho_{3}, 0}^{0, \rho_{3}} \leq \phi_{p}(l) .
$$

Then boundary value problem (1.1) (1.2) has three positive solutions in $K$.

Suppose $(H 1)-(H 4)$ hold, and

(H6): there exist $\rho_{1}, \rho_{2}, \rho_{3} \in(0, N)$, with $\rho_{1}<\rho_{2}<\gamma \rho_{3}$ such that

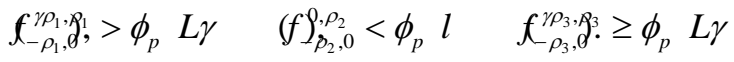

Then boundary value problem (1.1) (1.2) has two positive solutions in $K$.

Proof. Because the proof is similar to the proof of theorem in [9]. So we omit it here.

Corollary 4.1. Suppose $(H 1)-(H 4)$ hold. In addition, if there exists $0<\rho<N$ such that

$$
0 \leq f_{-\rho, 0}^{0}<\phi_{p}(l), \quad f_{-\rho, 0}^{\gamma \rho, \rho}>\phi_{p}(L \gamma), \quad 0 \leq f_{-N, 0}^{\infty}<\phi_{p}(l) .
$$

Then boundary value problem (1.1) (1.2) has three positive solutions in $K$.

Suppose $(H 1)-(H 4)$ hold, and if there exists $0<\rho<N$ such that

$$
\phi_{p}(L)<f_{0}^{-\rho, 0} \leq \infty, f_{-\rho, 0}^{0, \rho}<\phi_{p}(l), \phi_{p}(L)<f_{\infty}^{-N, 0} \leq \infty .
$$

Then boundary value problem (1.1) (1.2) has two positive solutions in $K$.

It is similar to the conclusion of Theorem 4.1 we obtain the following theorem

Theorem 4.2. Suppose $(H 1)-(H 4)$ hold, and if one of the following conditions holds:

(H9): There exist $\rho_{1}, \rho_{2} \in(0, N)$ with $\rho_{1}<\gamma \rho_{2}$ such that

$$
f_{-\rho_{1}, 0}^{0, \rho_{1}} \leq \phi_{p}(l), \quad f_{-\rho_{2}, 0}^{\gamma \rho_{2}, \rho_{2}} \geq \phi_{p}(L \gamma),
$$

(H10): There exist $\rho_{1}, \rho_{2} \in(0, N)$ with $\rho_{1}<\rho_{2}$ such that

$$
f_{-\rho_{1}, 0}^{\gamma \rho_{1}, \rho_{1}} \geq \phi_{p}(L \gamma), \quad f_{-\rho_{2}, 0}^{0, \rho_{2}} \leq \phi_{p}(l) .
$$

Then boundary value problem (1.1)(1.2) has a positive solution in $K$.

\section{REFERENCES}

[1] D. Guo, "Multiple Positive Solutions on Nonlinear Integral Equations and Applications," Applicable Analysis, Vol. 23, No. 1-2, 1986, pp. 77-84. http://dx.doi.org/10.1080/00036818608839632

[2] X. He, W. Ge and M. Peng, "Multiple Positive solutions 
for One-Dimensional p-Laplacian Boundary Value Problems,” Applied Mathematics Letters, Vol. 15, No. 8, 2002, pp. 937-943.

http://dx.doi.org/10.1016/S0893-9659(02)00067-8

[3] R. Y. Ma and D. M. Cao, "Positive Solutions to an m-Point Boundary Value Problem," Applied Mathematics-A Journal of Chinese Universities, Vol. 17, No. 1, 2002, pp. 24-30.

http://dx.doi.org/10.1007/s11766-002-0021-y

[4] B. Liu and J. S. Yu, "Solvability of Multi-Point Boundary Value Problems at Resonance(I)," India J. Pure Appl. Math., Vol. 33, 2002, pp. 475-494.

[5] J. Henderson and H. Wang, "Positive Solutions for Nonlinear Eigenvalue Problems," Journal of Mathematical Analysis and Applications, Vol. 208, No. 1, 1997, pp. 252-259. http://dx.doi.org/10.1006/jmaa.1997.5334

[6] R. Ma and N. Castaneda, "Existence of Solutions of Non- linear m-Point Boundary Value Problems," Journal of Mathematical Analysis and Applications, Vol. 256, No. 2, 2001, pp. 556-567. http://dx.doi.org/10.1006/jmaa.2000.7320

[7] D. Guo and V. Lakshmikantham, "Nonlinear Problems in Abstract Cones,” Academic Press, New York, 1988.

[8] D. Ma, Z. Du and W. Ge, "Existence and Iteration of Monotone Positive Solutions for Multipoint Boundary Value Problems with p-Laplacian Operator," Computers \& Mathematics with Applications, Vol. 50, No. 5-6, 2005, pp. 729-739.

http://dx.doi.org/10.1016/j.camwa.2005.04.016

[9] Y. Wang and C. Hou, "Existence of Triple Positive Solutions for Multi-Point Boundary Value Problems with a One-Dimensional p-Laplacian,” Computers \& Mathematics with Applications, Vol. 54, No. 6, 2007, pp. 793807. http://dx.doi.org/10.1016/j.camwa.2006.10.038 